\title{
Survivor and parent engagement in childhood cancer treatment in Iran
}

\author{
Shirin Ahmadnia ${ }^{1}$, Atena Kamel Ghalibaf ${ }^{1}$, Saba Kamkar ${ }^{2}$, Zahra Mohamadzadeh ${ }^{2}$ and Mithra Ghalibafian ${ }^{3}$ \\ ${ }^{1}$ Allameh Tabataba i University, Tehran 1489684511, Iran \\ 2MAHAK Charity-Society to Support Children Suffering from Cancer, Tehran 1956993461, Iran \\ ${ }^{3}$ MAHAK Paediatric Cancer Treatment and Research Centre (MPCTRC), Tehran 1956993461, Iran
}

\section{Abstract}

In Iran (with a population of 83 million), 19,973 children were diagnosed with cancer from 2009 to 2019 (MAHAK Charity). This study was part of the International Society of Paediatric Oncology, Paediatric Oncology in Developing Countries Committee, 'Patient, Family and Stakeholder Engagement Task Force' engagement study in ten low- and middle-income countries in 2019. We documented childhood cancer survivors and parents' experiences and quality of engagement, including perceptions and expectations, during their cancer treatment journey in Iran. Fourteen in-depth interviews and three focus group discussions were conducted by three Iranian qualitative researchers with 29 participants: recent adolescent/young adult survivors (12-20 years), long-term survivors (21-30 years) and parents [36-61 years (six mothers and four fathers)] from diverse socio-economic and ethnic backgrounds. Data were recorded, transcribed and analysed, and then translated from Farsi into English.Participants' expectations included inter alia improved communication and information flow among the key stakeholders including doctors, nurses, parents and patients. A need for improved patterns of doctor-patient relationships and communication, more effective psychosocial support and the importance of peer support groups (for survivors and parents) were reported. Participants identified areas of satisfaction and dissatisfaction regarding their actual engagement and decision-making. The dominance of a medical-only approach instead of multidisciplinary approach in care, the shortage of psychosocial support, the lack of an integrated system for providing information and delivering a package of printed material about the treatment journey and the absence of patient navigator in childhood cancer care systems were among obstacles for successful engagement of patients and parents throughout their cancer journey.

Keywords: adolescent, physician-patient relationships, cancer survivors, parents, communication, engagement, Iran

\section{Introduction}

According to a model created by Ward et al [1], childhood cancer incidence in 2015 was estimated to be 397,000 worldwide. Atun et al [2] predict that between 2020 and 2050 there will be 13,659,000 new cases of childhood cancer globally and 2,509,000 in uppermiddle-income countries.

Correspondence to: Shirin Ahmadnia Email: ahmadnia@atu.ac.ir

ecancer 2021, 15:1220

https://doi.org/10.3332/ecancer.2021.1220

Published: $19 / 04 / 2021$

Received: 11/10/2020

Publication costs for this article were supported by ecancer (UK Charity number 1176307).

Copyright: (c) the authors; licensee ecancermedicalscience. This is an Open Access article distributed under the terms of the Creative Commons Attribution License (http:// creativecommons.org/licenses/by/3.0), which permits unrestricted use, distribution, and reproduction in any medium, provided the original work is properly cited. 
In Iran, an upper-middle-income country with a population of 83 million, 19,973 children were diagnosed with cancer within the last 10 years based on data from the Society to Support Children Suffering from Cancer (MAHAK Charity), corresponding to the findings in a study conducted by Shabani et al [3]. These statistics roughly represent national figures, since MAHAK supports almost all children with cancer throughout the country. According to a recent Iranian study, the crude incidence of childhood cancer (0-14 years) was 16.8 per 100,000 for males and 16.56 per 100,000 for females [4].

Iran borders seven countries: Iraq, Turkey, Armenia, Azerbaijan, Turkmenistan, Afghanistan and Pakistan. It is a multi-ethnic nation with major groups, including Persians (61\%), Azerbaijanis (16\%; Azeris) and Kurds (10\%) [5]. It is estimated that there are approximately 1 million Afghan migrants and refugees living in Iran [6]. Iran's Gini coefficient was 40.8 in 2017 [7] and the gross domestic product per capita in 2017 was USD 5,520.3 [8].

Children and adolescents with cancer in Iran are treated in 32 hospitals under the supervision of medical sciences universities and governmental medical centres across the country. Efforts have been made to create universal healthcare in the country, but it is not yet realised [9]. Childhood cancer treatment costs are covered by a number of healthcare insurance companies and non-governmental organisations (NGOs). According to a recent study in Iran, families with a child with cancer are generally referred to insurance organisations and NGOs for financial support since they face substantial financial challenges [10]. MAHAK Charity is an NGO founded in 1991, and supports all children (ages 0-16) with cancer in Iran.

Families of children/adolescents with cancer worldwide face multiple difficulties, endure negative experiences and face many challenges. Edwards and Greeff in South Africa concluded that throughout the process of treatment, poor standards of information delivery and lack of cancer knowledge provision by the healthcare system personnel and a lack of patient-centred care have been reported to be of great concern for parents [11]. In Romania, researchers found that 'provision of information is a fundamental step towards shared decision-making. It enables minor patients and families to frame personal values when making decisions' [12]. The importance of an efficient doctor-patient relationship is well documented in the literature on health communications and sociology of medicine [13]. A study in Malawi reported that guardians felt reluctant to ask the nurses and doctors questions, because they were afraid that they would be told off or they were shy [14]. On the contrary, according to a study in the US, parents whose children were at the end of life were reported to be quite satisfied with the availability of healthcare providers and the adequacy of information received about their child's diagnosis and symptom management [15].

There are two sides of the therapeutic relationship, patients and their parents and healthcare professionals (doctors mainly, but also nurses, psychologists and social workers). In the current study, we focused only on one side - patients and their parents. This study was conducted as part of the International Society of Paediatric Oncology, Paediatric Oncology in Developing Countries Committee, 'Patient, Family and Stakeholder Engagement Task Force' study in low- and middle-income countries in the summer of 2019. One common finding across all project countries was communication issues between healthcare personnel and parents and patients, among family members including extended family members. The authors of this article documented survivors and parents' experiences of treatment engagement, perceptions and expectations, level and quality of engagement, particularly communication, during a child/adolescent's cancer treatment journey in Iran.

\section{Methods}

This qualitative study included a total of 29 participants representing: a) 'adolescent and young adult survivors', b) 'long-term survivors' and c) 'parents' from across the country. We held three focus group discussions (FGDs) and 14 in-depth interviews (IDIs). Three FGDs were held with a total of 15 participants divided as follows: a) seven adolescent and young adult survivors, b) three long-term survivors and c) five parents. Fourteen IDIs with additional participants (distinct from those in the FGDs) were conducted with the following: a) five adolescent and young adult survivors, b) five long-term survivors and c) four parents (see Table 1 for eligibility criteria).

The authors defined a sampling framework to achieve sociocultural diversity among a range of ethnicities in Iran. MAHAK Charity supports families of children with cancer in all 40 centres across Iran. Participant recruitment and access was realised through the MAHAK Charity database and archives of patient records using administrative support and reflecting MAHAK Charity's nationwide coverage. (Table 2 shows the socio-demographic characteristics of the study participants.) 
Table 1. Sample characteristics of participant eligibility criteria and study sample.

\begin{tabular}{|l|l|l|}
\hline \multicolumn{1}{|c|}{ Groups of participants } & \multicolumn{1}{|c|}{ Potential intended samples } & \multicolumn{1}{c|}{ Study samples } \\
\hline Adolescent/young adult survivors & $\begin{array}{l}\text { Adolescent and young adult survivors who had completed treatment }<2 \\
\text { years }\end{array}$ & 12 recent survivors (12-20 years) \\
\hline Longer-term survivors & $\begin{array}{l}\text { Longer-term survivors (all treatment being completed before the age of 18) } \\
\text { and }>5 \text { years incident free }\end{array}$ & 7 longer-term survivors (21-30 years) \\
\hline Parents & Parents as care givers during their child's treatment occurring < 5 years & 10 parents (36-61 years) \\
\hline
\end{tabular}

Table 2. Socio-demographic characteristics of the study participants.

\begin{tabular}{|c|c|c|c|c|c|}
\hline \multicolumn{6}{|c|}{ Adolescent/young adult survivors } \\
\hline & Participant pseudonym & Gender & Age in years & Ethnicity & Iran region \\
\hline 1. & Patient 1 & Male & 14 & Persian & Center \\
\hline 2. & Patient 2 & Male & 17 & Persian & Center \\
\hline 3. & Patient 3 & Female & 12 & Arab & South \\
\hline 4. & Patient 4 & Female & 20 & Kurd & Center \\
\hline 5. & Patient 5 & Male & 20 & Azari & Center-rural area \\
\hline 6. & Patient 6 & Male & 17 & Persian & Center \\
\hline 7. & Patient 7 & Male & 15 & Lur & West \\
\hline 8. & Patient 8 & Female & 17 & Persian & Center \\
\hline 9. & Patient 9 & Female & 15 & Persian & Center \\
\hline 10. & Patient 10 & Male & 16 & Persian & Center \\
\hline 11. & Patient 11 & Female & 15 & Persian & South \\
\hline 12. & Patient 12 & Female & 13 & Afghan & Center \\
\hline \multicolumn{6}{|c|}{ Longer-term survivors } \\
\hline 1. & Patient 13 & Male & 28 & Persian & Center \\
\hline 2. & Patient 14 & Male & 21 & Kurd & Center \\
\hline 3. & Patient 15 & Male & 24 & Azari & Center \\
\hline 4. & Patient 16 & Female & 24 & Azari & Northwest \\
\hline 5. & Patient 17 & Female & 28 & Azari & Center \\
\hline 6. & Patient 18 & Male & 23 & Persian & Center \\
\hline 7. & Patient 19 & Male & 30 & Persian (Mazani) & North \\
\hline \multicolumn{6}{|c|}{ Parents } \\
\hline 1. & Parent 1 & Female & 38 & Azari & Center \\
\hline 2. & Parent 2 & Female & 41 & Persian & Center \\
\hline 3. & Parent 3 & Male & 36 & Azari & Center \\
\hline 4. & Parent 4 & Male & 44 & Persian & Center \\
\hline 5. & Parent 5 & Male & 61 & Afghan-immigrant & Center-rural Area \\
\hline 6. & Parent 6 & Female & 39 & Persian & Center \\
\hline 7. & Parent 7 & Female & 44 & Azari & Center \\
\hline 8. & Parent 8 & Female & 33 & Persian & East \\
\hline 9. & Parent 9 & Female & 35 & Azari & Center \\
\hline 10. & Parent 10 & Female & 38 & Persian & Center \\
\hline
\end{tabular}


Potential participants from the capital city and other provincial cities were solicited to travel to the interview site in Tehran by MAHAK's social work department; participants' transport costs were provided by MAHAK. All participants agreed to take part in IDIs and FGDs and signed a participant consent form in Farsi after being made aware of the study's purposes.

All IDI and FGDs were recorded, transcribed in Farsi and content analysis was carried out to identify codes by three expert qualitative researchers (all authors of this paper: SA, AK, and ZM). Data analysis in this study focused on the survivors and parents' engagement during cancer treatment.

\section{Results}

Participants were recruited from diverse ethnicities, regions and genders to represent the multicultural context of Iranian society. (See Table 2 for the socio-demographic characteristics of the study participants.)

The results are categorised in to four main themes: 1) participant expectations, 2) nature and extent of actual engagement, 3) degree of satisfaction with the nature and level of engagement, and 4) degree of engagement affecting overall care experiences.

\section{Participant expectations}

The parents and survivors' expectations from the medical team were described and characterised as their 'ideal' doctor/nurse and parent/ patient relationship. The participants expected doctors to be good listeners, medical experts and experienced, responsive, having friendly attitudes, kind, sincere, accessible, patient, providing hope, up to date and sensitive to the pain and problems of their patients. Their expectations of nurses were to be expert (especially with regard to phlebotomy), experienced, with a friendly attitude, kind, sincere, understanding, patient, with good manners, giving hope and encouragement. Patient 13, a 28-year-old survivor of Hodgkin disease said:

Doctors need to spend much more time [with their patients]. They should explain everything well for the patient. They should give the information in simple understandable words. Doctors are looking down on patients. At last, much is depending on his experience and expertise.

Patient 14, a 21-year-old male who survived rhabdomyosarcoma, said, 'the nurses treated children kindly. They were patient enough for this job. Nurses should be kept responsible for what they do'. Parent 4, a 44-year-old father of a child who survived glioma mentioned: 'the family expects the care team to guide them more on what they should do and how to deal with the problems'.

\section{Nature and extent of actual engagement}

Decision-making on treatment options, symptom management and/or pain management options included the parents' lack of ability and control to decide on changes in the treatment or to change the hospital where the child was being treated. As parent 6, a 39-year-old mother of a survivor of non-Hodgkin lymphoma, said: 'We had to rely on the doctor's decisions. We could only decide about financial issues. We were not supposed to get involved in other matters'. Most of the parents were inactive when it came to making decisions and were receptive towards their child's doctors' decisions. Parents mentioned feeling powerless. They even made reference to when medical staff made wrong decisions that negatively impacted their child. The following quotation shows the attitude belonging to most parents. Parent 3, a 36-year-old father of a survivor of neurofibromatosis, stated: 'We believed that we shouldn't bother doctors and not to cause them headaches. My wife and I believed in and had faith in our doctor. We accepted whatever he told us because he had graduated and was an expert in this field so we trusted him'.

It was common for parents to avoid disagreement or protest when communicating with healthcare professionals and no participant reported taking legal action against a healthcare professional even when they had witnessed something going wrong. Patient 16 , a 24 -year-old female survivor of acute lymphoblastic leukemia (ALL) told the following story: 
Once, they injected me a wrong medicine, then I complained afterwards that I got a terrible stomach ache. They did not take my complaint seriously since I was only a kid at the time. Later however they found out that due to that mistake, my kidneys failed and they transferred me to ICU (The interviewer asked if her parents protested against the person in charge in the hospital following that occurrence and she said no, they never even thought of something like that).

Some parents reported they were able to make decisions. One example of a rare active decision-making was Patient 1, a 14-year-old survivor of ALL who spoke about a time his mother took self-reliant action:

First, I was under the supervision of a doctor who cared for me a lot. After a while, though, my mother searched a bit, got consultation from other people and found out another doctor in the same hospital was more of an expert. She decided to make a change in who to treat me. I then got transferred to a second doctor. Having learned this change, the first doctor got upset and told me: 'This is not fair. You are being unkind to me. I liked you so much and I would rather have you under my supervision. Why should your mother turn to the other doctor!'

Parent 10, a 38-year-old mother of a survivor explained her bad experience when requesting a CT for her child at the time and her reaction:

I told the doctor: Could you please take a CT scan of my child's lung? 'No Madam, why are you disturbing your child [by demanding this]? There is nothing to worry about'. I insisted: If there is nothing to worry about, then please do as I wish because it will be a relief to me. He finally reluctantly agreed with the CT scan after I insisted, and it was only then that we realised there was actually a mass in my child's lung....

\section{Necessity of a medical/psychosocial supportive care team}

Several respondents spoke about their difficulty in accessing doctors, nurses and the supportive care team. They expressed that these healthcare professionals were not reachable or were inaccessible. Patient 13, introduced earlier, stated:

My mother usually had to run after doctors and it was a tough situation especially when I was going through a difficult phase that is, when my illness relapsed. As an example, I remember they came to the ward to visit us, but the doctors could not stay long being too busy. My mother had to run after them in the stairs to be able to pose her questions.

Patient 15, a 24-year-old male survivor of a small round cell tumour, on the contrary, spoke positively about the psychosocial team's good accessibility (i.e., psychologist and social worker). 'Social workers were there to support mothers. They came to visit the mothers to encourage them. They made sure the mothers fully understood the situation'.

However, for communication of medical information and education about the child/adolescent's cancer, particularly during diagnosis, parents mentioned that the doctors were not completely transparent. The doctors used jargon while delivering information, which had a negative effect on the quality of communication. Thus, parents described using tactics to compensate the lack of information and education, e.g., tactics such as searching on the Internet or using peer group consultation. According to Parent 9, a 35-year-old mother of a survivor of neuroblastoma:

The doctor should be transparent and clear in announcing the illness. We usually do not understand what doctors say [medical jargon]. A doctor should talk to us in simple words according to and based on our level of knowledge and understanding.

Parent 1, a 38-year-old mother of a survivor of neuroblastoma explained:

We begged whoever [personnel] we had access to, behind the operation room, in order to ask for any information such as the surgeon's words about the operation and what was going on with our child's health there. Sometimes we took a copy of the reports being transferred from one department to the other. Most of the times, however, the information we got was of no use, because the terminology used was complicated and in English, so we could not understand anything! We therefore, usually asked other parents with earlier similar experiences, for example, we asked them 'what does "calcification" mean?' At the time, not many people had smart-phones to be able to search easily for unknown things to come to an answer. 
On the other hand, some parents stated that they were intentionally avoiding detailed information due to their fear of diagnoses of cancer or because of denial. Parent 5, a 61-year-old father of a survivor of medulloblastoma explains:

Whenever we asked any questions the doctor did answer us as much as we wanted. But we often did not inquire ourselves; i.e., we were afraid of asking too much because in that case the doctor had to tell us much of the details and the negative facts which we would rather not hear or learn.

The parents' sociocultural background impacted their communication with the medical team in terms of the discrimination they experienced as Patient 17, a 28-year-old female survivor of rhabdomyosarcoma mentioned:

According to the city/town from which patient's family came and/or their educational background, they received different levels of attention and services from the care team [that is, discriminatory]. Doctors had a better behaviour towards a child whose parents were e.g. lawyers, or they didn't care much when explaining things to parents who were less educated or from a lower social class; Doctors assumed that those parents cannot understand much, therefore they did not speak to them with details or for long.

\section{The degree of satisfaction with the nature and level of engagement}

The dissatisfaction with the medical team was mostly reported due to a lack of information provided by doctors about treatment procedures and/or disease. However, it was also related to how doctors broke bad news and/or nurses and doctors not being responsive (answering questions) or clear enough. Parent 10, a 38-year-old mother of a survivor of ALL related a negative experience:

I told my doctor that I am really angry with you. You are not telling me anything. I do not care if sometimes you play with my child, make humour or you are being nice to my child. What matters to me is to know what's really going on with my ill child! The doctor replied that I am not supposed to put you in a stressful situation by disclosing the details of the treatment.

A survivor of osteosarcoma, 17-year-old Patient 8, shared her positive experience:

There was a nurse who brought me homemade food to cheer me up. Sometimes she gave me gifts, some hair pins or lip balms. I was eagerly waiting to see her. Some of nurses helped me with my school home-works and let me sit in their room with them to have fun. They were kind to me. This nice behaviour helped me not to think of hospital as a bad and horrible place.

Parent 3, introduced earlier, shared a story about experiencing discrimination because of his Afghan ethnicity: 'Since we are Afghan migrants, we were not treated respectfully and in a decent manner in a hospital. If we were Iranians, they would not disrespect us that way. However, at the hospital in Tehran we received respect and there were no discriminations'.

Financial aspects of treatment are a main concern for the families. Families and survivors were happy with the financial support they received from NGOs and therefore experienced the elimination of a financial relationship with the doctors. Patient 15 , introduced earlier, said:

We heard that adult patients with cancer had to sell everything they owned to pay the treatment costs. There were ill people leaving the treatment due to not being able to pay the costs. However, this charity offers financial support for treatment and even provides financial support for university tuition fees for recovered patients.

\section{Degree of engagement affecting the overall care experience}

Parents and survivors spoke about the care experiences they had had with the medical team (i.e., doctor/nurses) and some mentioned the doctor's lack of sympathy and empathy. Patient 3, a 12-year-old female survivor of medulloblastoma said, 'I would have liked to open up to my physician and enjoy his sympathy. If the physician would have talked to me, giving me hope, I would have recovered earlier than I did'. Patient 19, a 30-year-old survivor of ALL mentioned: 'Doctors suppose emotional support and showing affections are not considered their duties. This is why they kept their distance from us'. 
Patient 18, a 23-year-old male survivor of a central nervous system tumour spoke about the positive and negative relationships with the psychosocial support team and shared the following: 'Parents need to be trained by psychologists to learn how to treat their children throughout the stages of the illness. This could have positive impact and influence children's mental health'.

Parent 2, a 41-year-old mother of a survivor of a primitive neuroectodermal tumor (PNET), spoke positively about the multidisciplinary team approach in treatment journey:

Physicians, nurses, social workers and psychologists along with each other and with a good cooperation can achieve optimal outcomes during treatment. We understand that nurses may not be capable of practicing some stuff, such as showing kindness and necessarily being companionate (due to work overload), therefore we need social workers who could be nice to children and talk to them. And as for parents, the presence of psychologists would be really helpful and effective.

The parents mentioned the necessity for an integrated system for providing information and delivering a package of printed material for parents, right in the beginning of the treatment journey and throughout $(A-Z)$. They wanted the company of someone in the healthcare centre just to guide them step by step in every phase. Patient 4, a 20-year-old female survivor of a germ cell tumour, explained:

There was no plan or systematic anticipation to inform and teach families about the illness details and the treatment process at the beginning of the hospitalisation. I suppose this is very important. It could at least ease and comfort families a little bit. It's important to inform families, e.g. about the side effects of the treatment beforehand.

\section{Discussion}

For the successful engagement of parents and patients with the medical team throughout the cancer treatment journey, our participants felt the need to be fully informed about the disease and treatment procedure details in simple understandable words and asked for a better relationship with the caregiving team. They believed this would build a positive and effective communicative relationship with the medical team, especially since doctors play a crucial role in this, despite the fact that it is often neglected in the country. Zamanzadeh et al [16] investigated factors affecting the communication between patients with cancer and nurses in Iran. In order to communicate with cancer patients effectively, they found that changes in the 'philosophy and culture of the care environment' and focusing on 'holistic and patient-centred' care are essential, Effective communication was also found to be essential for paediatric cancer treatment in a study conducted in the US, where 'clear and empathic delivery of diagnostic and prognostic information positively impacts the ways in which patients and families cope' [17]. These findings are similar to our study results.

The lack of proper communication between parents and patients and the medical teams, the dominance of a medical-only approach instead of a multidisciplinary approach in childhood cancer care and the absence of paediatric psychology support were found to be among the most important obstacles for the successful engagement of patients and parents throughout their cancer journey. These issues were also found to be problematic in India, where Singh et al [18] identified 'several barriers to communication such as paternalism in medicine, inadequate training in communication skills, knowledge of the grieving process, special issues related to care of children, and cultural barriers'. In Kenya, Njuguna et al [19] emphasised that paediatric oncology doctors needed to improve their communication skills. In regard to adolescents with cancer in Switzerland, Essig et al [20] found that their communication needs are rarely met. They noted that differential expectations regarding good communication by doctors and nurses should be considered when developing communication skills courses for oncology professionals.

A multidisciplinary approach was suggested by our participants, wherein they mentioned the need for the presence of a psychologist in addition to the social worker to sit in the room when the doctor broke the bad news of their child's cancer diagnosis. The parents stated that this interdisciplinary harmony needs to be developed and reinforced throughout the treatment journey. They stated that they were happy with the doctor's expertise, but for psychological pain and fear, they needed a specialist's help and support; this is something a psychologist would provide, but the doctors usually are not capable of or do not care about the same. Keramatinia et al [21] in Iran concluded that a multidisciplinary approach inter alia was associated with lower risk of mortality and improved survival of children with various cancer diagnoses. 
Carlson et al [22] also found that 'a multidisciplinary approach supported clinical efficiency and fostered seamless patient-centred care' for childhood cancer survivors in the US.

Parents required clear and consistent explanations about their child's disease and treatment procedure. This need is partly recognised and mentioned in 'the patient's bill of rights', which is usually written on a paper in a frame hanging on a wall at the hospital for the patients to see, but is not practiced actually or effectively. As a solution or to substitute the lack of adequate information and communication, which would increase their engagement in the treatment, the participants in our study suggested the provision of peer support groups for parents and patients. Some of them actually played and still play such a supportive and informative role for new patients in the hospitals. In this way, they compensate for what is lacking in the current (inadequate) relationship among doctors and patients/parents. For example, experienced parents would interpret the doctors' words for the parents of a sick child just hospitalised, and/or explain what parents should do whenever the child's condition became acute or how to manage pain etc. These peers would give advice based on their own experiences.

Therefore, when we asked parents and survivors for their feedback on potential areas for a better engagement, they suggested forming committees consisting of peer groups with the same experiences. They spoke of the formation of groups such as survivor or parent support committees. This is congruent with the findings of a study by Smith et al [23] in the US, who referred to the need for social support groups among adolescents and young adults with cancer. Swedish researchers Ljungman et al [24] also suggested 'facilitating access to social support systems as an important aspect of psychological help to parents of children on cancer treatment'.

The participants in our study also recommended appointing a position in the hospital for a person to be in charge of guiding and accompanying new diagnosed patients and families through wards of hospitals (i.e., patient navigator). Schroeder et al [25] stated that 'oncology patient navigation programmes have been successfully implemented in hospitals throughout the US enhancing the quality of cancer care'. A pilot patient navigation programme in Tanzania was established by those researchers 'to assist patients and their families with care coordination throughout the diagnosis and treatment process'. Their results suggest that even in a resource-limited setting, navigation programmes are a reasonable strategy for tackling barriers to care delivery and treatment abandonment, thus improving survival.

A comprehensive information package available at the beginning of the childhood cancer treatment journey (following a cancer diagnosis) could be provided for parents and patients as guidance on the child/adolescent's disease and relevant treatment procedures. The information would cover physical, mental and social guidelines, tips and advice for frequently asked questions and highlight the parents' role for improved involvement and engagement during the treatment journey. This would not require high costs and budgets could be covered under the routine treatment costs, while exploiting the support provided by social workers in the hospitals.

Elimination of the financial relationship between families and doctors, which is seen as an important factor for a better engagement of the families with the hospital medical teams (to include all potential patients and not only the higher socio-economic classes), is already on the agenda of the Ministry of Health. This has been partially achieved through the implementation of the new Health Reform Plan launched a few years ago with the purpose of realising social justice and reducing families' out-of-pocket health and care costs. Rarely hospitals in Iran have realised this approach. The participants in this study who had accessed this opportunity and received free services have expressed their high satisfaction about this.

\section{Conclusion}

As we explored the engagement quantity and quality of parents and survivors, we realised that parents were concerned about the lack of proper communication not only with physicians, but also with nurses, psychologists and social workers, and expressed a need for direct contact and dialogue. This will most likely require training in communication skills during diagnosis, investigations and treatment. In Iran, it is not common among healthcare professionals to function as a multidisciplinary team, which according to our findings is assumed to be necessary by patients with cancer and their parents. Implementation of a multidisciplinary approach in the childhood cancer treatment journey with an emphasis on the presence of psychosocial supportive team alongside the medical care team, especially when breaking bad news, is concluded to be necessary. This approach is feasible in Iran, with simple revisions in healthcare policies made at the level of the Ministry of Health guidelines and protocols or in every hospital if the director could be convinced that this would result in better healthcare outcomes. Written material for families about childhood cancer is another important communication tool for improved engagement and should be developed. 
Continued exploration of patient and parent engagement during childhood cancer treatment across Iran is warranted. Implementation of participant recommendations and evaluation of these changes will reveal the effectiveness of the changes in practice. Patient and parent engagement is a critical part of childhood cancer care and must be prioritised and respected.

\section{Acknowledgments}

We are especially thankful for the efforts made by Ms. Behnaz Asangari (psychologist, the head of MAHAK support services department); MAHAK psychologists Ms. Maryam Mohammadali and Mr. Reza Mashayekhi; MAHAK social workers Ms. Soraya Sabzian and Ms. Marzieh Bashiri; MAHAK international relations department experts Ms. Sepideh Esmaili, Ms. Farzaneh Sadeghi Nik and Ms. Roghayeh Hasani Tabar; and MAHAK public relations expert Ms. Zohreh Hashemi. We are also highly grateful to the survivors and parents who kindly shared their stories of their cancer treatment journey with us.Last but not least, we deeply appreciate the great support and opportunity we received from SIOP [Société Internationale d'Oncologie Pédiatrique (International Society of Pediatric Oncology)] PODC (Paediatric Oncology in Developing Countries) with special thanks and deep gratitude to Dr. Julia Challinor.

\section{Funding}

The authors would like to thank MAHAK Charity for its financial support for the research project, administrative support and provision of access to the participants for IDIs and FGDs.

\section{Conflicts of interest}

Saba Kamkar and Zahra Mohamadzadeh hereby declare their association as full-time employees of MAHAK Charity-Society to Support Children Suffering from Cancer and Mithra Ghalibafian hereby declares her association as full-time employee of MAHAK Paediatric Cancer Treatment and Research Centre (MPCTRC). Shirin Ahmadnia and Atena Kamel Ghalibaf, who voluntarily participated in this study, hereby declare that they have no conflict of interest of any kind.

\section{References}

1. Ward JZ, Yeh JM, and Bhakta M, et al (2019) Estimating the total incidence of global childhood cancer: a simulation-based analysis Lancet Oncol 20(4) 483-493 https://doi.org/10.1016/S1470-2045(18)30909-4 PMID: 30824204

2. Atun R, Bhakta N, and Denburg A, et al (2020) Sustainable care for children with cancer: a Lancet Oncology Commission Lancet Oncol 21(4) e185-e224 https://doi.org/10.1016/S1470-2045(20)30022-X PMID: 32240612

3. Shabani M, Saeedi Moghaddam S, and Ataeinia B, et al (2020). Trends of national and subnational incidence of childhood cancer groups in Iran: 1990-2016 Front Oncol 91428 https://doi.org/10.3389/fonc.2019.01428

4. Hassanipour S, Fathalipour M, and Delam H, et al (2019) The incidence of childhood cancer in Iran: a systematic review and metaanalysis, Iran J Ped Hematol Oncol 9(3) 193-206

5. Sawe BE Largest Ethnic Groups in Iran 2019 [https://www.worldatlas.com/articles/largest-ethnic-groups-in-iran.html]

6. Salavati B (2020) Iran migration outlook (Tehran: Danesh Bonyan Fannavar)

7. The World Bank GINI index (World Bank estimate) - Iran, Islamic Republic [https://data.worldbank.org/indicator/SI.POV. GINI?locations=IR] 
8. The World Bank GDP per capita (current US\$) - Iran, Islamic Rep [https://data.worldbank.org/indicator/NY.GDP.PCAP.CD?locations=IR]

9. Sajadi HS, Ehsani-Chimeh E and Majdzadeh R (2019) Universal health coverage in Iran: where we stand and how we can move forward Med J Islam Repub Iran 339 PMID: 31086788 PMCID: 6504945

10. Mojen L, Rassouli M, and Eshghi P, et al (2018) Pediatric palliative care in Iran: applying regionalization of health care systems Asian Pac J Cancer Prev 19 1303-1311 PMCID: 6031829

11. Edwards LB and Greeff LE (2017) A descriptive qualitative study of childhood cancer challenges in South Africa: thematic analysis of 68 photovoice contributions S Afr J 1(0) a14 https://doi.org/10.4102/sajo.v1i0.14

12. Badarau DO, De Clercq E, and Wangmo T, et al (2016) Cancer care in Romania: challenges and pitfalls of children's and adolescents' multifaceted involvement J Med Ethics 42 757-761 https://doi.org/10.1136/medethics-2016-103418 PMID: 27697792

13. Sisk BA, Mack JW, and Ashworth R, et al (2018) Communication in pediatric oncology: state of the field and research agenda Pediatr Blood Cancer 65(1) e26727 https://doi.org/10.1002/pbc.26727

14. Israels $\mathrm{T}$, Chirambo $\mathrm{Ch}$, and Caron $\mathrm{H}$, et al (2008) The guardians' perspective on paediatric cancer treatment in Malawi and factors affecting adherence Pediatr Blood Cancer 51 639-642 https://doi.org/10.1002/pbc.21703

15. Gilmer MJ, Foster TL, and Bell CJ, et al. (2013) Parental perceptions of care of children at end of life Am J Hosp Palliat Care 30 53-58 https://doi.org/10.1177/1049909112440836

16. Zamanzadeh V, Rassouli M, and Abbaszadeh A, et al (2014) Factors influencing communication between the patients with cancer and their nurses in oncology wards Indian J Palliat Care 20(1) 12-20 https://doi.org/10.4103/0973-1075.125549 PMID: 24600177 PMCID: 3931236

17. Blazi LJ, Cecchini C, and Habashy C, et al (2018) Communicating effectively in pediatric cancer care: translating evidence into practice Children 540 https://doi.org/10.3390/children5030040

18. Singh RK, Raj A, and Sujata P, et al (2015) Role of communication for pediatric cancer patients and their family Indian Journal of Palliative Care 21338 https://doi.org/10.4103/0973-1075.164888 PMID: 26600703 PMCID: 4617042

19. Njuguna F, Mostert S, and Seijffert A. et al (2015) Parental experiences of childhood cancer treatment in Kenya Support Care Cancer 23 1251-1259 https://doi.org/10.1007/s00520-014-2475-x

20. Essig S, Steiner C, and Kuehni C, et al (2016) Improving communication in adolescent cancer care: a multiperspective study Pediatr Blood Cancer 63(8) 1423-1430 https://doi.org/10.1002/pbc.26012 PMID: 27081706 PMCID: 5917069

21. Keramatinia AA, Mohseny M, and Akbari M, et al (2018) Determinants of survival of common childhood cancers in Iran J Res Med Sci 23 $1-5$

22. Carlson CA, Hobbie WL, and Brogna M, et al (2008) A multidisciplinary model of care for childhood cancer survivors with complex medical needs J Pediatr Oncol Nurs 25(1) 7-13 https://doi.org/10.1177/1043454207311741 PMID: 18187596

23. Smith AW, Parsons HM, and Kent EE, et al (2015) AYA HOPE study collaborative group. Unmet support service needs and healthrelated quality of life among adolescents and young adults with cancer. 2013: the AYA HOPE study Front Oncol 3 309-317

24. Ljungman L, Cernvall M, and Grönqvist $\mathrm{H}$, et al (2014) Long-term positive and negative psychological late effects for parents of childhood cancer survivors: a systematic review PLoS One 9(7) e103340 https://doi.org/10.1371/journal.pone.0103340 PMID: 25058607 PMCID: 4110004

25. Schroeder $\mathrm{K}$, Alavi $\mathrm{F}$, and Johnston $\mathrm{H}$, et al (2019) Impact of a pilot patient navigator program on pediatric cancer outcomes in a low resource setting J Clin Oncol 37(15) e18152 https://doi.org/10.1200/JCO.2019.37.15_suppl.e18152 